\title{
Absolute Calibration of a Large-diameter Light Source
}

\author{
J.T. Brack ${ }^{a *}$ R. Cope ${ }^{a, b}$, A. Dorofeev ${ }^{a}$, B. Gookin ${ }^{a}$, J.L. Harton ${ }^{a}$, \\ Y. Petrov ${ }^{a}$, A.C. Rovero ${ }^{\dagger \dagger}$ \\ ${ }^{a}$ Colorado State University, Department of Physics, Fort Collins CO 80523, USA, \\ ${ }^{b}$ Now at Cornell University, Ithaca, NY, \\ ${ }^{c}$ Instituto de Astronomía y Física del Espacio (CONICET-UBA), Buenos Aires, Argentina \\ E-mail: brackecolostate.edu
}

\begin{abstract}
A method of absolute calibration for large aperture optical systems is presented, using the example of the Pierre Auger Observatory fluorescence detectors. A $2.5 \mathrm{~m}$ diameter light source illuminated by an ultra-violet light emitting diode is calibrated with an overall uncertainty of $2.1 \%$ at a wavelength of $365 \mathrm{~nm}$.
\end{abstract}

KEYWORDS: Large detector systems for particle and astroparticle physics; Detectors for UV, visible and IR photons; Optics

PACS: 95.55.Cs Ground based UV, optical and IR telescopes; 96.50.sd Extensive Air Showers; 95.55.Qf Photometric, polarimetric, and spectroscopic instrumentation

Subjects: Cosmic rays; Fluorescence detectors; Calibration

${ }^{*}$ Corresponding author.

${ }^{\dagger}$ Member of Carrera del Investigador, CONICET 


\section{Contents}

1. Introduction 目

2. Technique: Use of $1 / r^{2}$ attenuation of light

3. Limits of $1 / r^{2}$ validity for extended sources and detectors 3

母. Hardware

4.1 Drum 4

4.2 LED light source

4.3 Light source electronics

4.4 Uniformity of drum emission 7

4.5. Calibration reference standard

4.6 Dark Hall and Dark Box 8

5. Drum absolute intensity calibration

6. Systematic checks and uncertainties 12

6.1 Deviations of measured points from $1 / r^{2}$

6.2 Uncertainties

77. Conclusions

\section{Introduction}

The Pierre Auger Observatory has been designed to study the origin and the nature of ultra highenergy cosmic rays, which have energies above $10^{18} \mathrm{eV}$ [1]. The Observatory, located in Malargüe, Argentina, consists of two detector systems which provide independent information on Extensive Air Showers (EAS) initiated by cosmic ray interaction in the Earth's atmosphere. The Surface Detector (SD) is composed of over 1660 water Cherenkov detectors located on a triangular array of $1.5 \mathrm{~km}$ spacing covering an area of $3000 \mathrm{~km}^{2}$ measuring EAS secondary particles reaching ground level [2]. The Fluorescence Detector (FD) consists of 27 telescopes distributed in buildings on the periphery of the SD overlooking the array [3] 䧃].

The UV-nitrogen fluorescence light produced along the core of the particle shower in the air is registered by the FD on clear nights, while the SD operates continuously. Events observed by both FD and SD provide the link from the FD, which is absolutely calibrated, to provide the energy calibration [5] of the higher statistics data from the SD. The reconstruction of air shower longitudinal profiles and the determination of the total energy of reconstructed showers depends on the conversion of ADC counts from the FD to an absolute light flux at the aperture for each pixel. This 
conversion must be available for each observing night and for all wavelengths of the FD response range. To achieve this objective three different FD calibration procedures are performed [G]: relative, multi-wavelength $[0][8]$, and absolute calibrations. The measurement of the intensity of the light source used for the absolute calibration is discussed in this paper.

The calibrated $2.5 \mathrm{~m}$ diameter portable light source (referred to as the "drum" because of its appearance) is used at the FD apertures, providing uniform illumination to each pixel at a single wavelength [3]. The known flux from the light source and the response of the acquisition system give the required calibration for each pixel. The procedure we used previously to calibrate the drum at the laboratory has been outlined elsewhere [10][11]. The drum and electronics are designed to have a pulsed intensity approximately matching the intensity of a typical EAS at the FD. This light flux is too low to be measured directly by a photodiode in the lab by about a factor of 10,000, and too bright to be calibrated using PMTs and single-photon counting techniques. Our new procedure described here uses the $1 / r^{2}$ attenuation of a point source with distance to allow absolute calibration of a small light source at a short distance using the calibrated photodiode and to relate this intensity to that of the drum at large distance using a PMT.

In Secs. 1 and 3 we introduce the technique and its limitations due to finite sizes of the source and detectors. The new light source and controlling electronics are described in Sec. 6 . Details of the new procedure in the laboratory and results are presented in Sec. 5, and a discussion of uncertainties is given in Sec. 6 .

\section{Technique: Use of $1 / r^{2}$ attenuation of light}

The factor of about 10,000 needed to accommodate the difference in intensities required by the calibrated photodiode and the intensity level of the drum is achieved, conceptually, by the following steps: The drum light source is pulsed, and at some large distance (in practice about $15 \mathrm{~m}$ in the lab in Malargue) a PMT is used to record a histogram of the intensity. Next, a small-diameter light source (which we call the rail light source) is set up at this same distance, and the intensity is adjusted to give the same PMT histogram intensity as for the drum. Then, without changing its intensity, the rail light source is moved to an optical bench in a dark box where the pulsed absolute intensity can be measured directly using a calibrated photodiode at a short distance (in practice about $10 \mathrm{~cm}$ ).

The PMT is used only as a measure of the drum intensity relative to that of the rail light source, so no PMT calibration is required. The same LEDs and driving electronics are used for the drum and the rail light source, ensuring identical pulse characteristics.

In practice, several conditions and systematic checks are required to ensure reliability of the measurement. First, since the angle of incidence for a photon on the front glass PMT face and photocathode affects PMT response, the distance between the PMT and the drum light source must be sufficiently large that the drum provides light normally incident on the PMT (see Sec. [3). The $15 \mathrm{~m}$ distance limits the angle of incidence to less than $5 \mathrm{deg}$.

A potentially large source of error is reflection from the walls of the dark hall or from the interior of the optical bench dark box. Light blocking baffles were used in both environments. To confirm $1 / r^{2}$ behavior, the rail light source was moved along a rail in $1 \mathrm{~m}$ steps from about $10 \mathrm{~m}$ to $15 \mathrm{~m}$ distance from the PMT, and in $10 \mathrm{~cm}$ steps from about $1 \mathrm{~m}$ to $10 \mathrm{~cm}$ from the NIST-calibrated 
photodiode in the dark box. Stray light and reflections will in general not vary as $1 / r^{2}$, so this is an important test.

\section{Limits of $1 / r^{2}$ validity for extended sources and detectors}

The number of photons $N_{D}$ observed by a detector of radius $R_{D}$ emitted from a Lambertian disk of radius $R_{E}$ can be written:

$$
N_{D}=\frac{N_{E}}{2} \times\left(\left(1+\frac{r^{2}+R_{D}^{2}}{R_{E}^{2}}\right)-\sqrt{\left(1+\frac{r^{2}+R_{D}^{2}}{R_{E}^{2}}\right)^{2}-4 \frac{R_{D}^{2}}{R_{E}^{2}}}\right)
$$

where $N_{E}$ is a total number of photons emitted by the disk, and $r$ is the distance between emitter and the detector. Equation 3.1 assumes that the planes of the detector and emitter are perpendicular to the optical axis passing through the central points.

Two limits of Eqn. 3.1 are of interest here. For $r=0$ we obtain the following approximations:

$$
N_{D}=N_{E} \times \begin{cases}1 & \text { if } R_{D} \geq R_{E} \\ \frac{R_{D}^{2}}{R_{E}^{2}} & \text { if } R_{D}<R_{E}\end{cases}
$$

indicating that when the emitter is smaller than the detector all emitted photons are detected, and that if the emitter becomes larger than the detector the fraction of detected photons is proportional to the ratio of the areas. At the limit of large distances, $r \gg \sqrt{R_{D}^{2}+R_{E}^{2}}$, Eqn. 3.1 reduces to a description of a finite detector observing a point source:

$$
N_{D}=\frac{N_{E} \cdot R_{D}^{2}}{r^{2}} .
$$

Figure 1 shows the percentage deviation from $1 / r^{2}$ behavior for detector-source combinations corresponding to our equipment. The solid red line represents Eqn. 3.1 evaluated for the geometry used in the dark box, where the radii of the photodiode and the rail light source are $4 \mathrm{~mm}$ and $2 \mathrm{~mm}$, respectively, and the deviations from point-like $1 / r^{2}$ behavior vary from less than $1 \%$ to $0.05 \%$ at distances between 100 and $1000 \mathrm{~mm}$, as used in the dark box measurements. Similarly, the dashed magenta line is evaluated for the PMT-rail light source geometry, for which the radii are $37 \mathrm{~mm}$ and $2 \mathrm{~mm}$, and deviations are below $1 \%$ at distances of $1 \mathrm{~m}$, while the smallest distance using this combination in our measurements is $10 \mathrm{~m}$ for which approximately $0.01 \%$ deviation is calculated. The dot-dash blue line, for the PMT-drum combination, uses radii of $37 \mathrm{~mm}$ and $1250 \mathrm{~mm}$. At a distance of $15 \mathrm{~m}$, as used in the dark hall, the deviation is again less than $1 \%$. For all distances and geometry used in the calibration described here, deviations are well below $1 \%$. (See Fig. 8 for actual distances used.)

Radiant intensity of the emitter $I$ in units of $\left[\frac{p h o t o n s}{s r}\right]$ can be calculated from the number of emitted photons, $N_{E}$, using Eqn. 3.4:

$$
I(\Theta)=\frac{N_{E}}{\pi} \cdot \cos (\Theta)=I_{0} \cdot \cos (\Theta)
$$

where $\Theta$ is the angle from the normal to the surface of the emitter. Our measurement of the drum intensity results in $I_{0}$. 


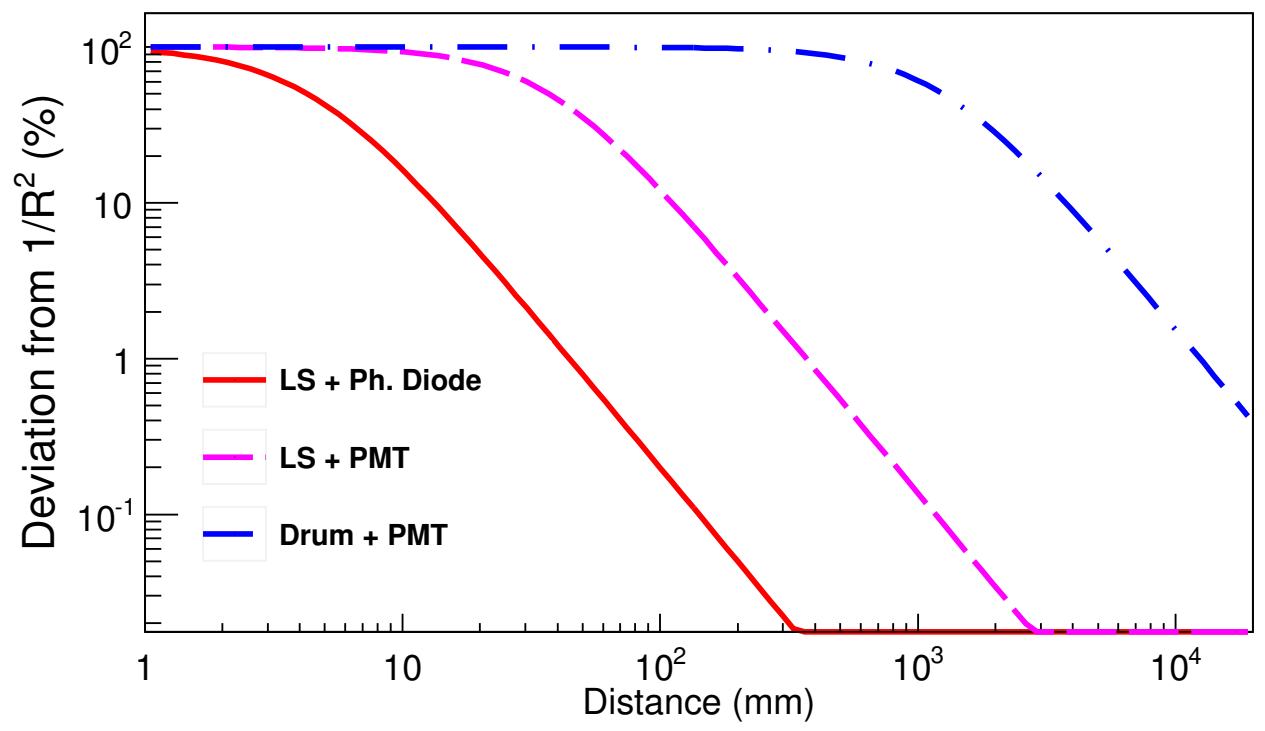

Figure 1. Deviation from $1 / r^{2}$ behavior as a function of distance between source and detector for the sizes (radii) of the sources involved in the procedure: drum $(1.25 \mathrm{~m})$, PMT $(37 \mathrm{~mm})$, and Light Source $(2 \mathrm{~mm})$.

\section{Hardware}

\subsection{Drum}

The portable light source (drum) has been designed to uniformly illuminate all 440 pixels in a single camera simultaneously. The drum is a cylinder of $2.5 \mathrm{~m}$ diameter and $1.4 \mathrm{~m}$ deep constructed in sections, using laminations of paper honeycomb core and aluminum sheet (see Fig. 2). The sides and back surfaces of the drum interior are lined with Tyvek ${ }^{\circledR}$, a material diffusively reflective in the UV. The front face of the drum is a $0.38 \mathrm{~mm}$ thick Teflon ${ }^{\circledR}$ sheet, which transmits light diffusively. We have developed a stabilized UV light source (see Sec. 4.2) that is placed on the front drum surface illuminating the interior so that the light experiences diffusive reflection from the Tyvek before being diffusively transmitted by the Teflon front surface of the drum. The multiple diffuse reflections result in a more uniform and Lambertian light source (see Sec. 4.4). This same light source is used on the rail with a different diffuser.

The FD apertures are $2.2 \mathrm{~m}$ in diameter, thus part of the $2.5 \mathrm{~m}$ diameter drum face is masked during use for FD calibration. During absolute calibration of the drum in the laboratory, described below, a $2.2 \mathrm{~m}$ diameter opaque mask is mounted on the front face of the drum, mimicking the aperture condition.

\subsection{LED light source}

The LED and associated components are contained in an aluminum box $(18 \times 15 \times 4.5 \mathrm{~cm})$, which is mounted directly on the face of the drum illuminating the interior. When configured as the rail light source, the same electronics box is mounted on the rail in the dark hall at varying distances from the PMT for calibration measurements. Different diffusers are used in the two configurations. 


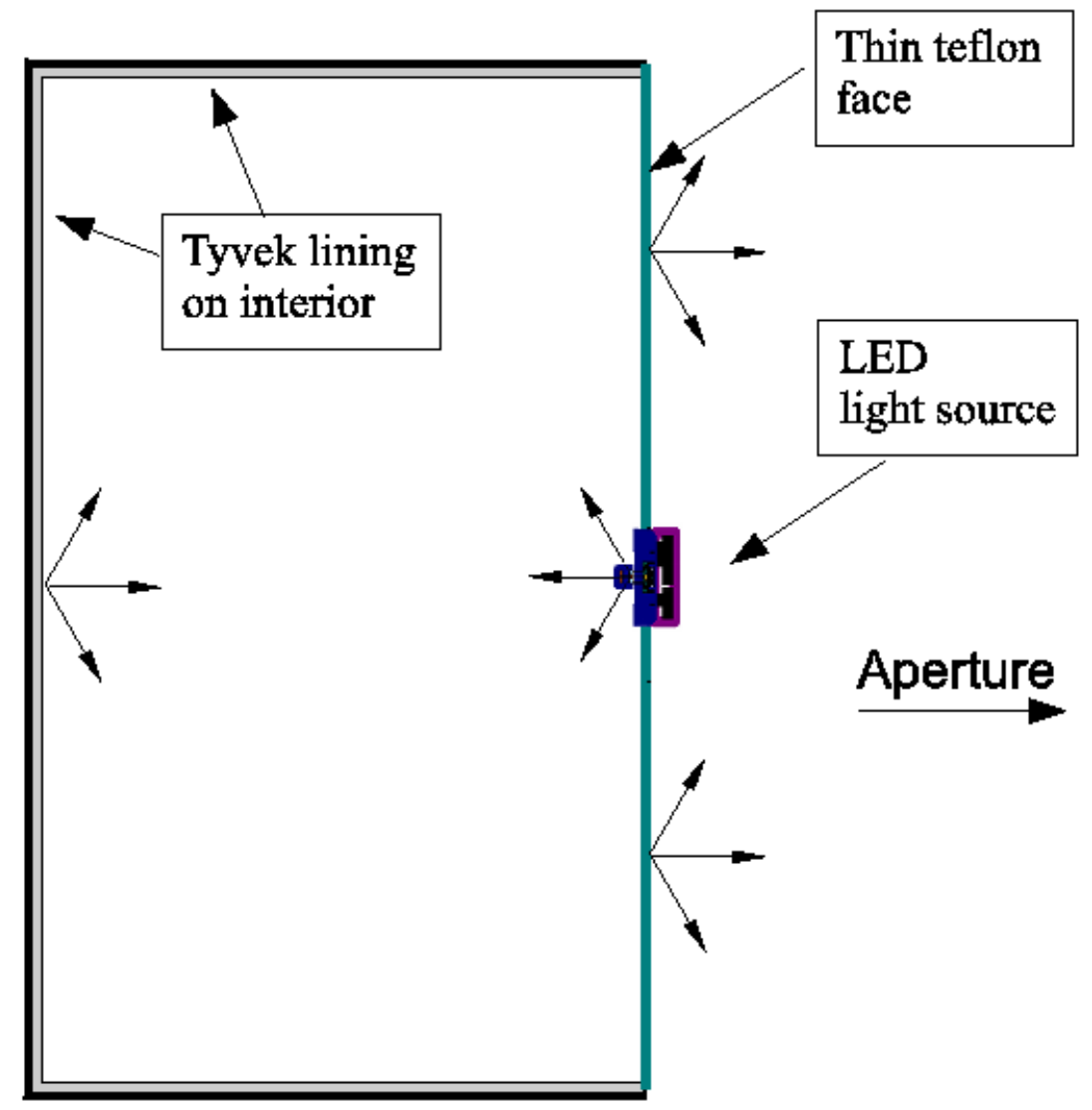

Figure 2. Schematic of the calibration light source and drum. The drum is $1.4 \mathrm{~m}$ deep and $2.5 \mathrm{~m}$ in diameter.

The LED is mounted on a Peltier device with a radiator and fan for temperature control; these components protrude from one side of the box. To enhance uniform distribution of photons, a Teflon diffuser covers the LED. For use in the drum, the diffuser is cylindrical. Photons exit radially, making at least one diffusive reflection from the Tyvek-lined reflector cup mounted inside the drum before hitting the inner surfaces of the drum. For use on the rail and in the dark box, this cylindrical diffuser is replaced with a $2.5 \mathrm{~cm}$ diameter Tyvek disk mounted in a $2.5 \mathrm{~cm}$ diameter, $2.5 \mathrm{~cm}$ long pipe. A $2.5 \mathrm{~cm}$ diameter black disk with a $4 \mathrm{~mm}$ diameter hole masks the Teflon at the exit of the pipe.

The light source, mounted in the reflector cup on the drum face, is shown in Fig. B, with the cylindrical diffuser in place.

\subsection{Light source electronics}

The electronics package includes a $600 \mathrm{MHz}$ BlackFin BF537 processor running $\mu$ Clinux, controlling a Xilinx Spartan3A programmable logic device on a separate board, which also holds four 12-bit DACs controlling LED current and two 12-bit ADCs for reading the PMT and light source 


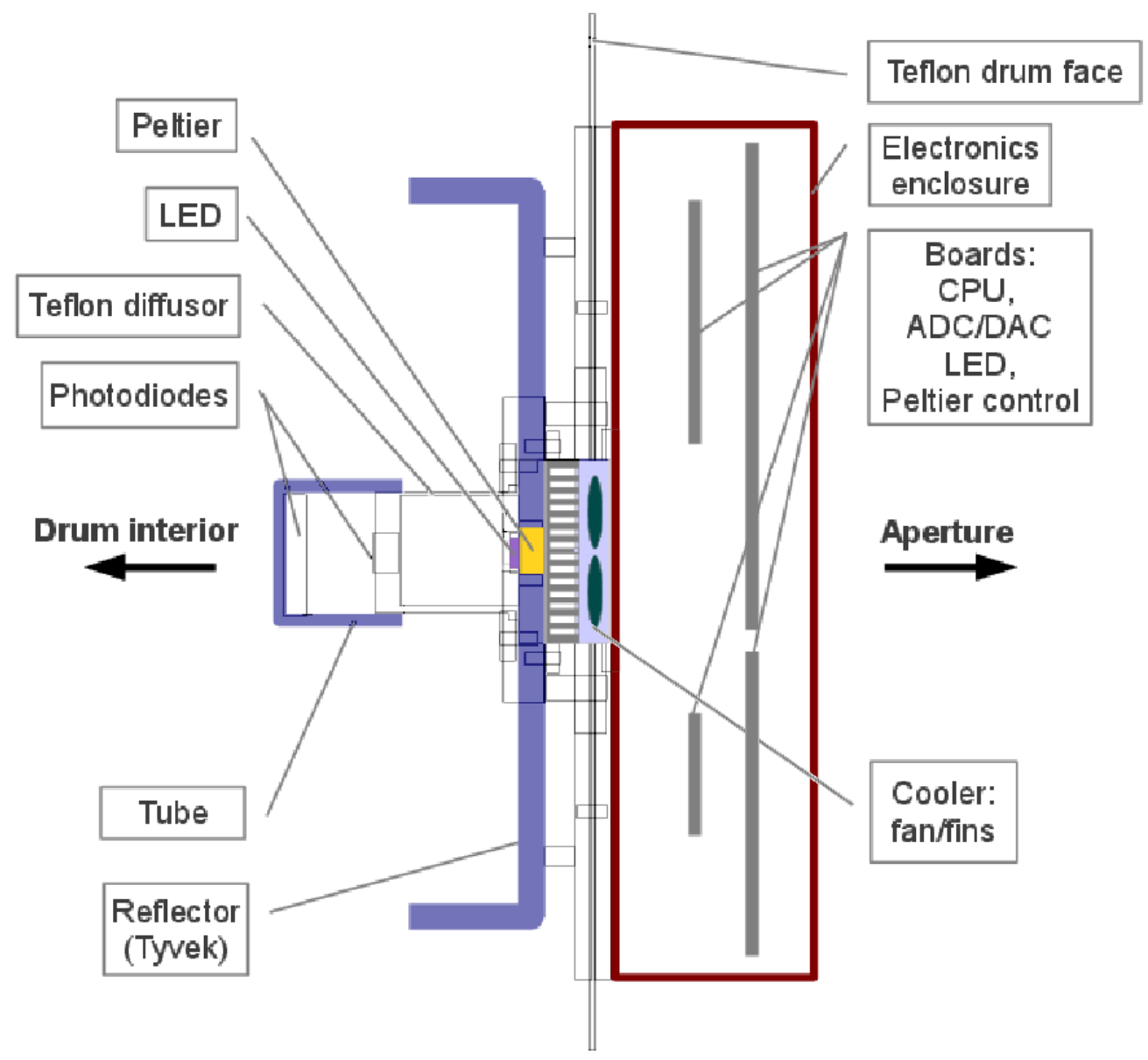

Figure 3. Schematic diagram of the light source assembly, mounted on the drum, showing the position of the temperature controlled LED, the Teflon diffuser and the monitoring silicon detector. The reflector cup is $15 \mathrm{~cm}$ in diameter, and is permanently mounted inside the drum.

monitoring photodiode signals. The DACs and ADCs run at $100 \mathrm{MS} / \mathrm{s}$. The linux OS runs cgi code controlling the light source pulse via specially designed web pages. Pulse shape, width, amplitude, and rate are programmable from the web page. Traces from the ADCs are stored in $32 \mathrm{Mb}$ onboard memory and are read out via ethernet connection.

Stability of the LED [12] junction temperature is crucial for maintaining constant optical output as a function of wavelength, and for stability over time Fig. $\rightarrow$ shows measurements of LED emission taken several days apart from a continuously pulsing LED. Tests at Colorado State University of high output LEDs run at large duty factors or high current have shown that the central wavelength can shift by as much as $10 \mathrm{~nm}$ accompanied by an enhanced tail in the long wavelength end of the output distribution, if insufficient temperature control is provided. To control junction temperature as much as possible, the LED is mounted directly on the surface of a Peltier device using an electrically non-conductive thermal paste. The Peltier is similarly mounted on a radiator. A dedicated microcontroller mounted on a separate board in the electronics package controls the Peltier current with feedback from a thermistor mounted at the LED. Under the pulsed current con- 


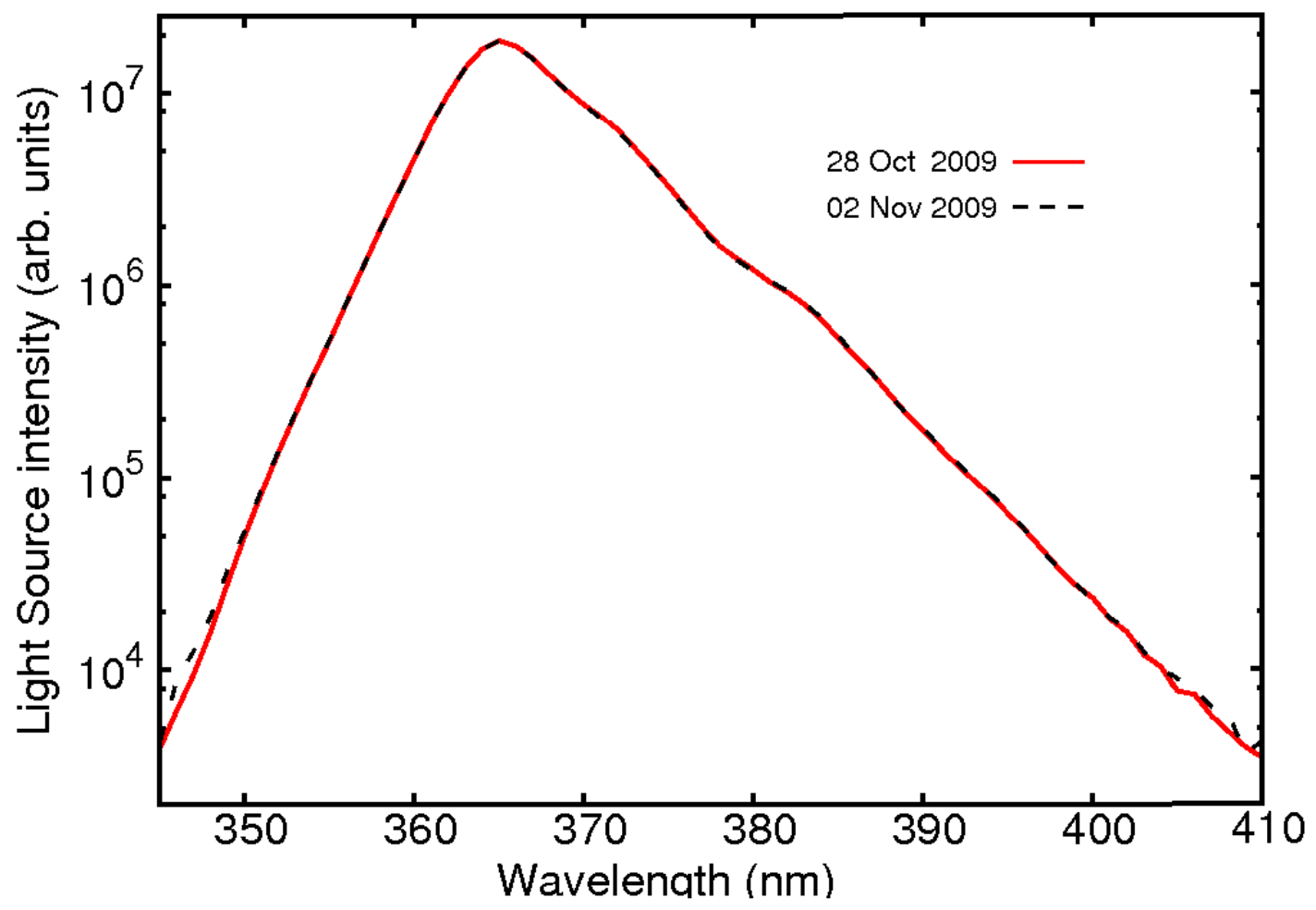

Figure 4. Spectral stability of the LED in the lab. The spectra were taken five days apart. The stability of total number of photons is better than $0.1 \%$.

ditions used for our measurements, the controlled LED surface temperature is stable at $22 \pm 0.5 \mathrm{C}$. This temperature and the ambient temperature in the electronics box are readout from the CAN bus.

\subsection{Uniformity of drum emission}

The drum light source has been constructed to provide a uniform flux of photons through the FD apertures during calibration. This is accomplished by using the diffusively reflecting and transmitting materials described in 4.1. Validity of these Lambertian properties of the drum can be found in [10] where the drum was viewed at angles from 0 to 25 degrees with a CCD and the change in surface intensity was negligible.

\subsection{Calibration reference standard}

The calibration reference is a UDT100 silicon photodiode [13], calibrated by NIST and available as a calibration standard [9], equipped with a $0.4999 \mathrm{~cm}^{2}$ mask. We use three such calibrated photodiodes for comparison of performance. The calibration of the photodiode at $365 \mathrm{~nm}$ is given by NIST as $0.119 \mathrm{~A} / \mathrm{W}$, with $\mathrm{k}=2$ relative expanded uncertainty of $1.6 \%$, corresponding to a $1 \sigma$ uncertainty of $0.8 \%$, provided the associated uncertainties in the NIST calibration measurements are normally distributed. 
The photodiode is read out using a Keithley 6485 picoammeter. Figure 5 shows the output of the picoammeter in Q-mode. Each time bin corresponds to an output cycle of the ammeter, corresponding to an integration period. Each step corresponds to a cycle during which the LED was pulsed at a typical intensity. The flat regions before and after the pulsing show the rate of integration of the photodiode dark current, which is on the order of $1 \mathrm{pA}$ for these $1 \mathrm{~cm}^{2}$ large-area photodiodes at room temperature.
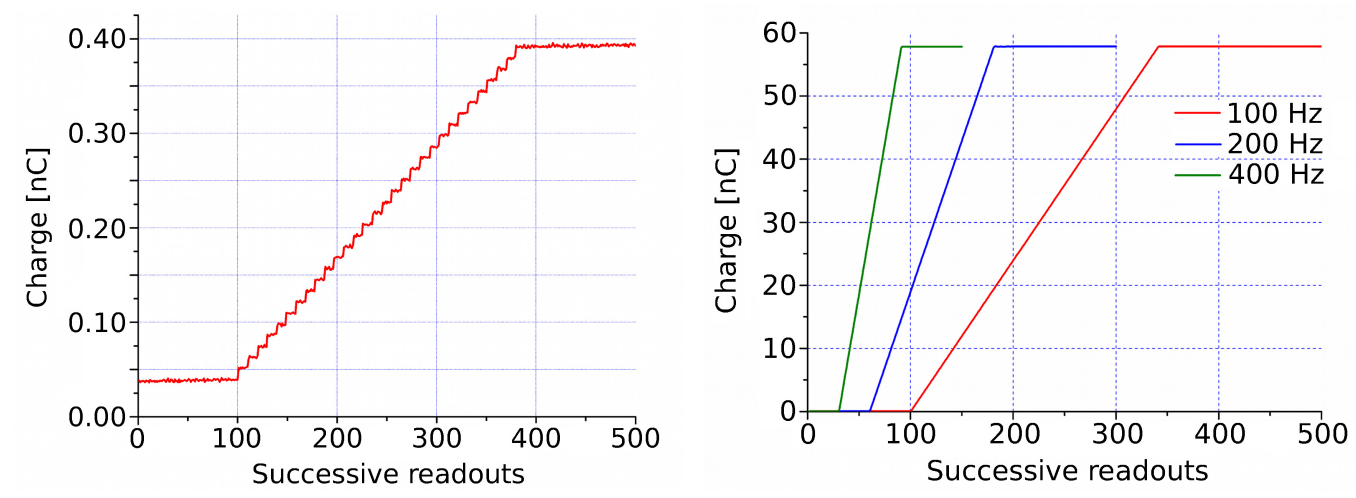

Figure 5. Integration of the NIST-calibrated photodiode current using the Keithley 6514 electrometer in Q-mode. Left: 30 pulses of the LED at the rate of $1 \mathrm{~Hz}$. The sampling rate is faster than $1 \mathrm{~Hz}$ so one can see clear steps when each pulse happens and the accumulated charge increases. Right: 5000 pulses submitted at different rates illustrate independence of the total collected charge from the pulsing rate.

\subsection{Dark Hall and Dark Box}

The dimensions of the dark hall are $4 \times 4 \times 17 \mathrm{~m}$. The layout is shown in Fig. 6. A $0.30 \mathrm{~m}$ diameter hole in the end wall allows the addition of a $0.6 \times 0.6 \times 1.5 \mathrm{~m}$ dark box in an adjoining room. Curtains at the mid-point of the dark hall create a light blocking baffle, preventing primary reflections from the walls, ceiling and floor from reaching the entrance to the dark box, which forms a second light baffle.

A cart mounted on a rail provides a movable mount for the rail light source, which can be positioned at points from 10 to $15 \mathrm{~m}$ from the PMT face.

In the dark box, a linear actuator positions the rail light source at points from $10 \mathrm{~cm}$ to $1 \mathrm{~m}$ from the calibration photodiode remotely, without opening the box. The centers of the drum, the rail light source, the PMT, and the photodiode are on-axis with the two baffles.

\section{Drum absolute intensity calibration}

Following the technique described above, we positioned a PMT in the dark box at a distance of $r_{D}=15.5 \mathrm{~m}$ from the drum (see the upper diagram in Fig. 6). Then a series of PMT traces was recorded, each corresponding to a single $5 \mu$ s pulse, and the average of the ADC counts per pulse $H^{\text {Drum }}\left(r_{D}\right)=H^{\text {Drum }}$ was measured (see the blue square on Fig. 8). Next, the light source was dismounted from the drum, the cylindrical Teflon diffuser was removed, and the $2 \mathrm{~mm}$ diffuser was installed in its place - thus forming the rail light source. The rail light source was then mounted 


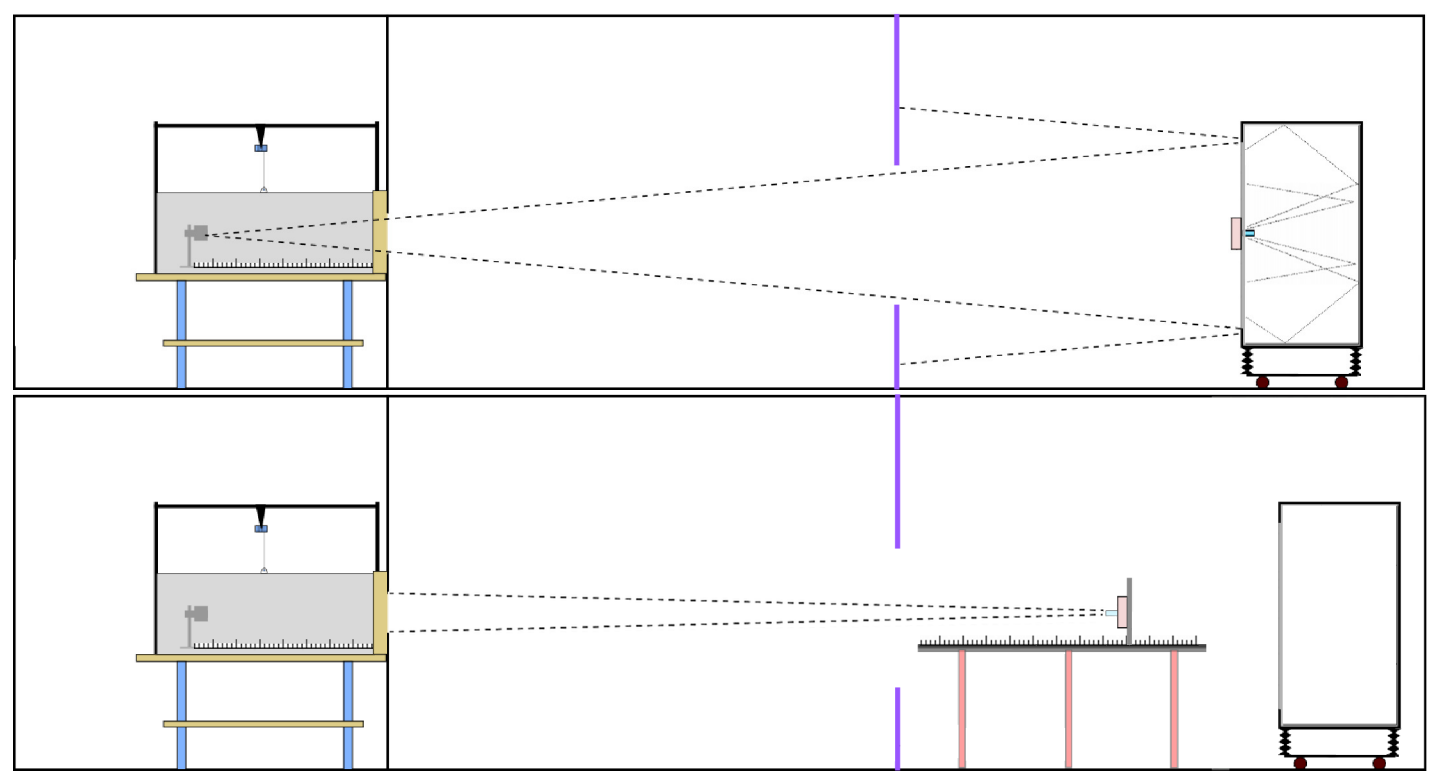

Figure 6. A schematic of the calibration dark hall and equipment. Top: as set up for drum intensity measurements. Bottom: as set up with the rail light source for measurements of its intensity before transfer to the dark box with the calibrated photodiode.

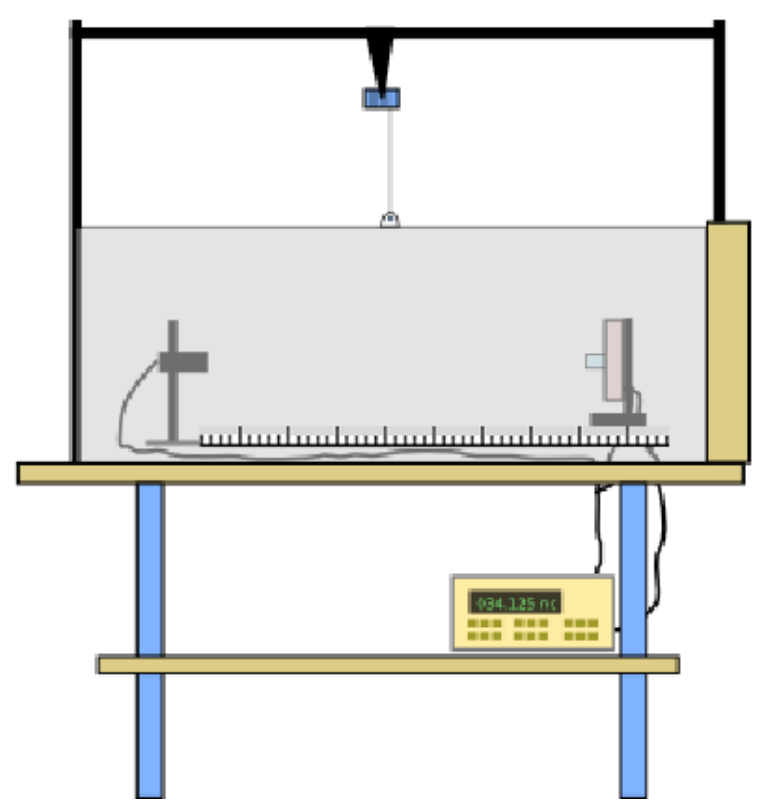

Figure 7. Schematic of the calibration dark box with rail light source and calibrated photodiode.

on the rail (see lower diagram in Fig. (6), and its intensity was adjusted to give a PMT response similar to that observed with the drum. A series of PMT traces was recorded, each corresponding to a single $5 \mu$ s pulse from the rail light source, and again the average per pulse was measured $H^{\text {Rail }}\left(r_{i}\right)=H_{i}^{\text {Rail }}$, but now at six different positions along the rail $r_{i}$ between 10.75 and $15.25 \mathrm{~m}$ 
from the PMT; the corresponding points are marked as red squares on Fig. 8. The rail light source was never placed at the full drum distance (the drum was physically in the way) and it was purposely set at a slightly lower intensity such that, as its position on the rail was varied, the response spanned the measured drum intensity. It is important that the PMT detection system is linear over the range of responses, as demonstrated in Fig. 8, and that the drum intensity is contained within the linear region. In principle, the drum could have been much farther away, provided these conditions were satisfied.

The rail light source was then moved into the dark box, and the PMT was replaced by the calibrated photodiode. The LED was flashed in the same manner as on the rail, and the charge per pulse $Q\left(r_{j}\right)=Q_{j}$ was measured, now in absolute units of $\left[\frac{C}{\text { pulse }}\right]$ with the photodiode dark current subtracted. We did this at several distances $r_{j}$ between $10 \mathrm{~cm}$ and $1 \mathrm{~m}$. These measurements are marked as black dots on Fig. 8.

In these measurements we have three combinations of light sources with detectors: drum with PMT; rail light source with PMT; and rail light source with calibrated photodiode. The LED has a non-zero width in wavelength of emission, and the diffusers can affect the spectrum from the drum and rail light source. Additionally, the PMT and photodiode have different wavelength-dependent responses. The convolution of the spectra of these three sources with the wavelength-dependent responses of the two detectors plays a role in the extraction of the drum intensity.

We define the response of a detector at a particular wavelength $\lambda$ to be $R_{D}(\lambda)=\frac{\operatorname{Signal}_{D}}{n_{D}(\lambda)}$ where Signal $_{D}$ is the readout of the detector exposed to some source (in ADC counts for the PMT; in Coulombs for the calibrated photodiode) and $n_{D}(\lambda)$ is the number of photons as a function of wavelength incident on a particular detector. We write the total number of photons incident on a detector over the source spectrum as $N_{D}$ in terms of detector response integrated over the source spectrum

$$
N_{D}=\frac{\text { Signal }_{D}}{\int_{\lambda} R_{D}(\lambda) \text { Spectrum }_{E}(\lambda) d \lambda}=\frac{\text { Signal }_{D}}{\Omega_{D}^{E}}
$$

where $\operatorname{Spectrum}_{E}(\lambda)$ is the normalized emission spectrum of the source $E$. Thus $\Omega_{D}^{E}$ is a constant for each source-detector combination which depends on the wavelength-dependence of the source emitter and detector. We calculate these constants using our measurements of the optical properties of the diffusive properties of the materials and the bare spectrum of the LED.

The drum radiant intensity $I_{0}^{\text {Drum }}$, in units of $\left[\frac{p h o t o n s}{s r \times p u l s e}\right]$, can then be written using equations Eqns. 3.3, 3.4, and 5.1 as

$$
I_{0}^{\text {Drum }}=\frac{N_{E}^{\text {Drum }}}{N_{E}^{\text {Rail }}} I_{0}^{\text {Rail }}=\frac{H^{\text {Drum }} \cdot r_{D}^{2}}{\left\langle H_{i}^{\text {Rail }} \cdot r_{i}^{2}>\right.} \frac{\Omega_{P M T}^{\text {Rail }}}{\Omega_{P M T}^{\text {Drum }}} \times \frac{<Q_{j} \cdot r_{j}^{2}>}{A \cdot \Omega_{\text {Photodiode }}^{\text {Ral }}}
$$

where $N_{E}^{\text {Drum }}$ and $N_{E}^{\text {Rail }}$ are the total number of photons emitted by the drum and rail light sources, respectively, $I_{0}^{\text {Rail }}$ is the radiant intensity of the rail light source, and $A$ is the area of the photodiode (mask area and uncertainty provided by NIST). The wavelength dependence is integrated over in the three numbers $\Omega_{P M T}^{\text {Rail }}, \Omega_{P M T}^{\text {Drum }}$, and $\Omega_{\text {Photodiode }}^{\text {Rail }}$. As one can see from Eqn. 5.2, the detector responses appear as $($ Response $) \cdot(\text { Distance })^{2}$, which are constants for each source and detector combination (assuming $1 / r^{2}$ behavior), and therefore we take the averages of these constants over the different distances for the rail light source and for the single distance for the drum measurements. 


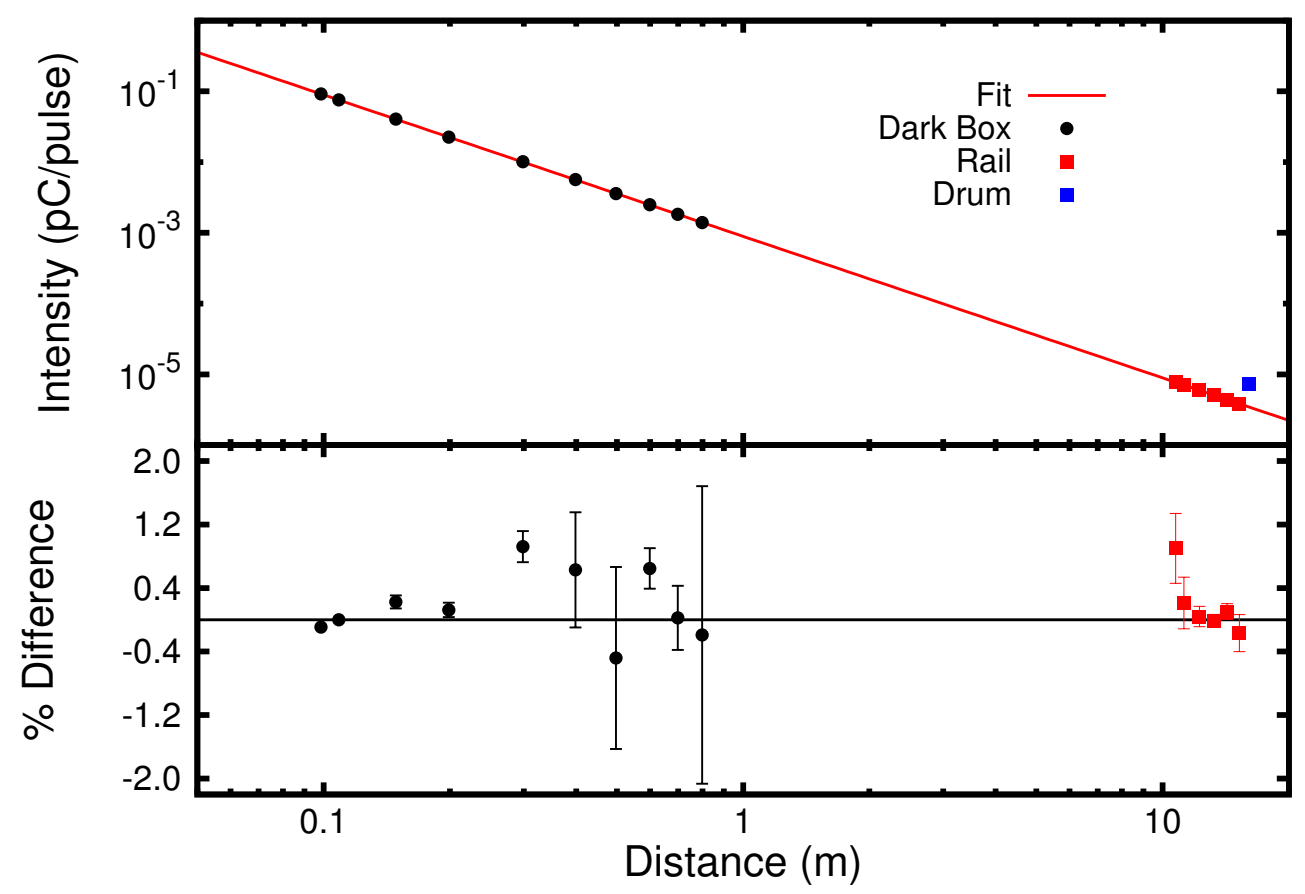

Figure 8. Measurements of PMT and photodiode responses to the light sources, and differences from a $1 / r^{2}$ fit (red line). The fit is made to only the black points, corresponding to measurements using the calibrated photodiode. Red points represent measurements using the PMT with the light source on the rail, and are normalized as a group to the fit. The blue point is the PMT measurement of the drum intensity with the same PMT normalization factor applied. The residuals are shown on the bottom axis as percentage difference from the $1 / r^{2}$ fit.

The response of the NIST calibrated photodiode sets the absolute intensity scale in units of $[C]$ for all the PMT responses. A ratio of the photodiode response to the PMT response in units of $\left[\frac{C}{A D C \text { count }}\right]$ can be written as

$$
\frac{<Q_{j} \cdot r_{j}^{2}>}{<H_{i}^{\text {Rail }} \cdot r_{i}^{2}>}
$$

This conversion factor is then applied to all PMT measurements $\left(H_{i}^{\text {Rail }}\right.$ and $\left.H^{\text {Drum }}\right)$ individually. The upper portion of Fig. 8 shows a graphical representation of this process. Each point has uncertainties $\frac{\sigma}{\sqrt{N}}$ on the mean of $\mathrm{N}$ repeated measurements, and are too small to be seen on this plot.

Eqn. 5.2 has four natural factors:

$$
F_{1}=\frac{H^{\text {Drum }} \cdot r_{D}^{2}}{\left\langle H_{i}^{\text {Rail }} \cdot r_{i}^{2}>\right.} ; F_{2}=\frac{\Omega_{P M T}^{\text {Rail }}}{\Omega_{P M T}^{\text {Drum }}} ; F_{3}=<Q_{j} \cdot r_{j}^{2}>; F_{4}=\frac{1}{A \cdot \Omega_{\text {Photodiode }}^{\text {Ral }}}
$$

$F_{1}$ is calculated from the dark hall measurements with the PMT; it has systematic effects due to measurements of the distance between PMT and the drum or rail source; reflections and stray light; misaligned pointing of the rail light source; and PMT stability. $F_{2}$ is calculated based on the PMT quantum efficiency and the source spectra of the drum and rail light source; if the PMT QE is not 
flat over the two spectra and the two source spectra are not identical, then a systematic effect is introduced. $F_{3}$ is calculated from the dark box measurements using the calibrated photodiode; it may have systematic effects due to photodiode calibration; reflections in the dark box; and distance measurements from the rail light source to the photodiode. $F_{4}$ is calculated from the NIST-provided calibration of the photodiode and the area of the photodiode mask, and our measured emission spectrum for the rail light source.

A typical value calculated from a calibration curve taken in 2010 gives radiant drum intensity $I_{0}^{\text {Drum }}=5.70 \pm 0.12 \times 10^{8}\left[\frac{\text { photons }}{\text { sr.pulse }}\right]$

The possible sources of systematic error mentioned above are discussed in the next section.

\section{Systematic checks and uncertainties}

The distance between the drum or the rail light source and the PMT is known to a centimeter at worst; this uncertainty enters in the square of the distance. For the drum at $15.5 \mathrm{~m}$ this uncertainty of $0.01 \mathrm{~m}$ results in a $0.1 \%$ systematic on $I_{0}^{\text {Drum }}$. Similarly, the closest distance of the rail light source to the PMT $(10 \mathrm{~m})$ results in a conservative $0.2 \%$ uncertainty on $I_{0}^{\text {Drum }}$. In the dark box we measure the distance from the rail source to the photodiode to about $0.5 \mathrm{~mm}$, and this results in a $1 \%$ overall uncertainty.

We studied reflections in the dark hall by removing the anti-reflective baffles and covers on the floor, and we found no measurable change in the PMT response to the drum or rail source. Evidently the small entrance hole to the dark box provided effective baffling. Stray light and reflections could be indistinguishable from errors in distance measurements; we used a semi-analytic calculation to study this issue and are able to bound the effect of stray light to $0.1 \%$ on $I_{0}^{\text {Drum }}$.

If the rail light source luminous surface were not perpendicular to the optical axis, say, while on the rail, or had a different angle to the axis in the dark box, then a systematic effect results. Mechanical measurements limit this angle to about 4 degrees, resulting in a systematic uncertainty on the drum intensity of $0.6 \%$.

The time required to make a full calibration of the drum, including drum intensity, rail light source work with the PMT, and dark box measurements with the calibrated photodiode, is about three hours. The systematic due to PMT stability was estimated by making repeated measurements of the drum or rail source on a time scale of a several hours or on different days. The repeatability of the system (dominated by the PMT) is at the $0.4 \%$ level. The stability of the LED light source itself was discussed above, and is at the $0.1 \%$ level over a few days.

NIST provides an uncertainty on calibration of the photodiode $(0.8 \%)$ and on the uncertainty in the area of the mask for the photodiode $(0.1 \%)$.

To estimate the deviation of $F_{2}$ from unity, we measured, as a function of wavelength, the reflectivity of the Tyvek in the drum and the Teflon face of the drum and the transmission of the Teflon face. Convoluting these factors, allowing for several reflections in the drum before transmission through the face, results in an estimate of the drum emission spectrum. In the same way we calculated the spectrum of the rail light source. We convoluted these spectra against the PMT quantum efficiency, and we find $F_{2}=1.001$. We do not make this small correction, rather we quote a $0.1 \%$ systematic uncertainty. 
Similarly the convolution of the rail light source spectrum with the calibrated photodiode is uncertain due to both the uncertainty in the photodiode calibration and the rail light source spectrum. But in this case, since the absolute calibration of the NIST-provided calibration appears alone (not in a ratio as in $F_{2}$ for the PMT measurements) we quote the full $0.8 \%$ NIST uncertainty.

The calibration of the Keithly electrometer is uncertain at the $1 \%$ level, provided by the manufacturer.

\subsection{Deviations of measured points from $1 / r^{2}$}

Shown in the lower portion of Fig. \& are the deviations of measurements taken at all distances with the $1 / r^{2}$ fit to the NIST points; the points from the rail source with the PMT are laid on the curve as discussed above. Deviations of the points from $1 / r^{2}$ are all below $1 \%$, mostly within the statistical uncertainties, and we observe no significant pull of the fit. The geometrical configurations of the dark hall and the dark box are potential sources of systematic uncertainties due to reflections from walls or blockage of direct line of sight from detectors to all points on the light source. We take the lack of a systematic deviation of points from $1 / r^{2}$ in Fig. 8 as confirmation that such effects are quite small.

\subsection{Uncertainties}

Table 1 lists the uncertainties associated with the absolute intensity calibration of the drum light source. Statistical uncertainties in the terms $\langle Q\rangle,\left\langle H_{L S}\right\rangle$ and $\left\langle H_{D}\right\rangle$ from Eqn. 5.2 are vanishingly small and are not listed. Repeated measurements of these terms are dominated by the drift in light source intensity and detector response, which are listed. The overall uncertainty in the calibration of the Auger Observatory Fluorescence Detectors involves additional factors such as temperature variations, reflections in the FD apertures and FD data analysis, which are not discussed here.

\section{Conclusions}

The measurement in the laboratory of the absolute intensity of the $2.5 \mathrm{~m}$ diameter drum calibration light source is the first step in the production calibration of the Auger Observatory fluorescence detectors. The sensitivity of the NIST-calibrated photodiode used as a calibration reference is some 4 orders of magnitude below that required to measure the drum intensity directly, forcing us to use some method of boosting the output to a measurable level for calibration. Working in a $17 \mathrm{~m}$ long dark hall allowing measurements at varying distances between the light source and detectors, we have established a simple procedure using the $1 / r^{2}$ reduction of light flux, eliminating use of neutral density filters on an optical bench as used previously for this reduction. The use of this technique has significantly reduced the dominant uncertainties from those related to use of the neutral density filters, leading to an overall uncertainty in the light source intensity of $2.1 \%$.

\section{Acknowledgments}

We acknowledge the contributions (and frustrations) of earlier collaborators in this Auger Observatory FD calibration effort, especially G. Hofman, R. Meyhandan, R. Knapik, and P. Bauleo. 
Table 1. Uncertainties for the drum intensity absolute calibration.

\begin{tabular}{clr}
\hline \hline & Quantity & Uncertainty (\%) \\
\hline Rail light source: & Stability & 0.1 \\
& Rail Light Source and Drum relative emission spectra & 0.1 \\
& Rail light source alignment & 0.6 \\
Distances: & Photodiode to rail light source & 1.0 \\
& PMT to rail light source & 0.2 \\
Stray light: & PMT to drum face & 0.1 \\
Photodiode: & NIST calibration @365 nm & 0.1 \\
& Active area & 0.8 \\
& Electrometer calibration & 0.1 \\
PMT : & Electrometer configuration repeatability & 1.0 \\
Total uncertainty & Stability & 1.0 \\
\hline \hline
\end{tabular}

\section{References}

[1] J. Abraham [Pierre Auger Collaboration], NIM A 523 (2004) 50-95.

[2] I. Allekotte, et al., for The Pierre Auger Collaboration, NIM A 586 (2008) 409.

[3] J Abraham et al. [Pierre Auger Collaboration], NIM A 620 (2010) 227-251.

[4] H. Klages, for The Pierre Auger Collaboration, Journal of Physics: Conference Series, 375 (2012) 052006.

[5] C. Di Giulio for the Pierre Auger Collaboration, Proceedings of the 31st ICRC, Lodz, Poland (2009); and R. Pesce for the Pierre Auger Collaboration, Proceedings of the 32nd ICRC, Beijing (2011).

[6] R. Knapik [Pierre Auger Collaboration], Proceeding 30 ${ }^{\text {th }}$ ICRC, Merida (2007).

[7] A. Rovero, et al., for The Pierre Auger Collaboration, Astroparticle Physics 31 (2009) 205.

[8] L. Wiencke, for the Pierre Auger Collaboration, Journal of Physics: Conference Series, 160 (2009) 012037.

[9] T.C. Larason, S.S. Bruce, and A.C. Parr, "Spectroradiometric Detector Measurements", National Institute of Standards and Technology, Calibration Program, Gaithersburg, MD 20899-2330, Special Publication 250-41, 1998.

[10] J. T. Brack, et al., Astropart. Phys. 20 (2004) 653.

[11] P. Bauleo [Pierre Auger Collaboration], Proceeding $29^{\text {th }}$ ICRC, Pune (2005), 8, 5-8.

[12] NSHU550 UV LED, Nichia America Corp., 48561 Alpha Drive, Wixom, MI 48393.

[13] UV100 photodiode, UDT Sensors, Inc, Hawthorne, USA. 